\title{
Complementary and Alternative Medicine: An Academic View
}

\author{
G.F. Gensini ${ }^{\mathrm{a}}$ D. Lippi ${ }^{\mathrm{b}} \quad$ A. Conti ${ }^{\mathrm{C}}$ A.A. Conti ${ }^{\mathrm{a}}$ \\ a Department of Critical Care Medicine and Surgery, University of Florence, and Don Carlo Gnocchi Foundation, \\ Departments of ${ }^{b}$ Anatomy, Histology and Legal Medicine, and ${ }^{c}$ Pathophysiology, University of Florence, \\ Florence, Italy
}

In the last 20 years international scientific literature has shown a steady increase in discussions concerning complementary and alternative medicine (CAM) [1]. When dealing with this subject, many issues arise. It is imperative to understand firstly the kind of medical reality concealed beyond the names of these practices and secondly, the problems posed to the medical profession by their increased therapeutic usage. Important questions further arise regarding the introduction of CAM therapies in medical curricula.

The reconstruction of the cultural context within which CAM has developed cannot escape a historical and linguistic reflection: a semantic analysis clearly indicates that this terminology is a well-defined historical product and conceals a specific conceptualisation that very often also involves a value judgement.

In 2000, the British House of Lords, through a select committee, commissioned research to illuminate the then current status of 'complementary therapies', in an attempt to understand the CAM therapies that could be considered real medical treatments [2]. The Commission classified therapies into three well-defined groups: Principle Disciplines, Complementary Treatments and Alternative Disciplines. The linguistic problem here revealed is substantial rather than formal. The Commission stated that it is first necessary to escape a terminology that involves the concept of 'alternative': this definition, which has been used in recent years, considers the practice of CAM as optional, justifying its use when scientifically sanctioned treatment is not available, or has failed.

In English, the terms 'alternative' and 'complementary' are mostly used, underlining a specific relationship with biomedicine. However, in France, CAM is known as 'médecines parallèles', with the precise intention of stressing the constant distance between orthodoxy and non-conventional medicine. Often, it is a negative definition such as 'not orthodox', 'not official' that is used highlighting what these medicines are not.

CAM treatments greatly differ from one another, but they have a common feature in that they often do not have their theoretical foundation in basic biological sciences. CAM therapies were very widely promoted in the late 19 th and early 20th centuries in many western countries, particularly North America. In the western world, their use is currently on the increase, especially in Englishspeaking societies. Recent data from the National Health Interview Survey (USA) indicate that in the 12 months previous to the interview, $33.5 \%$ of American women used CAM. Caucasian, elderly and educated individuals were more likely to use CAM [3]. Other recent data point

\section{KARGER}

Fax +4161306 1234

E-Mail karger@karger.ch

www.karger.com
C 2005 S. Karger AG, Basel

1011-7571/05/0146-0441\$22.00/0

Accessible online at:

www.karger.com/mpp
Andrea A. Conti

Dipartimento di Area Critica Medico Chirurgica

Università degli Studi di Firenze, Viale Morgagni 85

IT-50134 Firenze (Italy)

Tel. +39055 417928, Fax +39055 4379384, E-Mail aa.conti@dac.unifi.it 
to the fact that in this society, CAM therapies are increasingly adopted not only in benign acute transient conditions like colds and similar infections but also in dealing with serious chronic irreversible pathologies, such as neoplastic conditions. Such an increased use of CAM in the oncologic context in many cases has not yet been appropriately investigated by means of controlled clinical research.

CAM therapies propose a new relationship between patients and physicians, a holistic approach [4] to the patient and a re-evaluation of the psychosomatic component that include:

- An awareness that medicines may heal the disease but not the illness, which may very often have a psychic cause or component.

- A dissatisfaction with physicians and with the National Health Service.

- An increase in chronic diseases due to ageing, and those which cannot be satisfactorily treated with medicines.

- A fear of non-desired collateral or toxic effects.

- An increasing suspicion directed towards science.

- An interest in extra-European cultures, arising from ethnic modification in modern societies.

The availability of academic courses focusing on CAM is gaining ground. Most medical schools in the United States and Canada offer lectures in holistic medicine or CAM as well as postgraduate CAM conferences. Moreover, in many medical schools in North America courses related to CAM constitute a required part of the curriculum. For example, the University of Arizona College of Medicine offers a programme in integrative medicine with CAM, which is an attempt to provide a new model for medical education [5].

Medical schools must take note of the main reasons that lead patients to seek CAM, and develop a proper medical curriculum to include the understanding of patients' need for CAM. General practitioners may regularly face patient enquires about CAM. Consequently, they must acquire appropriate knowledge. Limited resources and institutional resistance together with the lack of evidence of CAM outcomes may pose barriers to this kind of education. However, in Italy a postgraduate course in Advanced and Integrated Medicine for general practitioners is provided in Umbria and public hospitalbased phytotherapy is provided in Empoli, Tuscany. Indeed, CAM is entering the Italian National Health System and many other initiatives have appeared recently. However, these efforts are based on individual initiatives and not on planned and shared programmes. Thus, the introduction of CAM into the curriculum remains a major issue in medical education. Medical schools must provide the guarantee of quality of information, knowledge management and continuing medical education, in order to escape the traps of quacks and incompetent and unpractised apprentices [6]. The Florence Medical School provides, for example, an integrated course in CAM in the second semester of the fifth year. It stresses the synthesis of humanistic education, patient- and relationshipcentred care, and deontological aspects of medicine, while taking into account historical, philosophical and anthropological perspectives on healthcare in different cultures. The foundation of a practical application of various medical systems - scientific evidence pertaining to CAM - is a major aspect of debate inasmuch as this medical school has long been engaged in the teaching of evidence-based medicine [7]. Since there is always the unavoidable problem of time constraint, students are offered an opportunity of going deeper into these subjects via optional courses of elective didactic activity. Thanks to this involvement of the Florentine University, the Italian centre for evidence-based medicine in Florence, and the Florentine College of Physicians in the provision of CAM-related elective didactic activity, the understanding and management of CAM is expected to have further important consequences from an economic and legal point of view. The application of the principles of evidence-based medicine to CAM should highlight the real outcomes of these therapies, underlining both risks and benefits. From an educational perspective, the learning outcomes for courses in CAM, such as the one implemented at the Florence Medical School, should be aimed at students acquiring critical evaluative tools, useful in assessing the profile of every applied and potentially usable CAM therapy. Teachers, however, are not called upon to teach CAM; they should teach 'about' CAM, meaning that the focus of CAM didactics should be on an assessment methodology shared between teachers and learners. Undergraduate students should acquire cognitive instruments in order to know, not practice, CAM therapies. They ought, at least, to know of the main ones so that at the end of the course they should be able to critically evaluate in an autonomous way the efficacy, safety, tolerability and manageability of commonly used CAM. In the Florence Medical School, teachers of the CAM course have different and integrative backgrounds (pharmacological, epidemiological, clinical, forensic, historical and ethical) with a common denominator - a profound knowledge of applied health methodology. 
The legal implications with regard to CAM and medical curriculum must also be considered. For example, the Italian law on drugs requires that the academic world take up a definite position on the sale of the over-thecounter CAM remedies. In this light, the Florence Medical School also provides its students with basic legal and forensic elements for the assessment of the (currently) correct use of CAM approaches that have the most reliable evidence basis: acupuncture, phytotherapy and manual medicine.
In conclusion, the spread of knowledge about CAM represents a further option for patients, who must be informed about these treatments with the same deontological precision as that used for traditional and scientific approaches.

\section{Acknowledgements}

The authors would like to thank Prof. Luisa Camaiora, B.A., M.Phil., for her correction of the English.

\section{References}

1 Murphy LS, Reinsch S, Najm WI, Dickerson VM, Seffinger MA, Adams A, Mishra SI: Searching biomedical databases on complementary medicine: the use of controlled vocabulary among authors, indexers and investigators. BMC Complement Altern Med 2003;3:3.

$\checkmark 2$ Complementary medicine: time for critical engagement. Lancet 2000;356:2023.
3 Upchurch DM, Chyu L: Use of complementary and alternative medicine among American women. Womens Health Issues 2005; 15:5-13.

4 Lewith GT, Breen A, Filshie J, Fisher P, McIntyre M, Mathie RT, Peters D: Complementary medicine: evidence base, competence to practice and regulation. Clin Med 2003;3:235240.

$\checkmark 5$ Maizes V, Schneider C, Bell I, Weil A: Integrative medical education: development and implementation of a comprehensive curriculum at the University of Arizona. Acad Med 2002; $77: 851-860$.
6 Ernst E: Complementary medicine: where is the evidence? J Fam Pract 2003;52:630-634.

7 Gensini GF, Conti A, Lippi D, Conti AA: Full integration of teaching 'Medical Humanities' in the medical curriculum: the challenge of the Florence Medical School. Med Princ Pract 2005; 14:64-65. 